\title{
A transgenic approach to study argininosuccinate synthetase gene expression
}

\author{
Shih-Chang Shiue ${ }^{1}$, Miao-Zeng Huang ${ }^{2}$ and Tsung-Sheng $\mathrm{Su}^{1,2^{*}}$
}

\begin{abstract}
Background: Argininosuccinate synthetase (ASS) participates in urea, nitric oxide and arginine production. Besides transcriptional regulation, a post-transcriptional regulation affecting nuclear precursor RNA stability has been reported. To study whether such post-transcriptional regulation underlines particular temporal and spatial ASS expression, and to investigate how human ASS gene behaves in a mouse background, a transgenic mouse system using a modified bacterial artificial chromosome carrying the human ASS gene tagged with EGFP was employed.

Results: Two lines of ASS-EGFP transgenic mice were generated: one with EGFP under transcriptional control similar to that of the endogenous ASS gene, another with EGFP under both transcriptional and posttranscriptional regulation as that of the endogenous ASS mRNA. EGFP expression in the liver, the organ for urea production, and in the intestine and kidney that are responsible for arginine biosynthesis, was examined. Organs taken from embryos E14.5 stage to young adult were examined under a fluorescence microscope either directly or after cryosectioning. The levels of EGFP and endogenous mouse Ass mRNAs were also quantified by S1 nuclease mapping. EGFP fluorescence and EGFP mRNA levels in both the liver and kidney were found to increase progressively from embryonic stage toward birth. In contrast, EGFP expression in the intestine was higher in neonates and started to decline at about 3 weeks after birth. Comparison between the EGFP profiles of the two transgenic lines indicated the developmental and tissue-specific regulation was mainly controlled at the transcriptional level. The ASS transgene was of human origin. EGFP expression in the liver followed essentially the mouse Ass pattern as evidenced by zonation distribution of fluorescence and the level of EGFP mRNA at birth. However, in the small intestine, Ass mRNA level declined sharply at 3 week of age, and yet substantial EGFP mRNA was still detectable at this stage. Thus, the time course of EGFP expression in the transgenic mice resembled that of the human ASS gene.

Conclusions: We demonstrate that the transgenic mouse system reported here has the merit of sensitivity and direct visualization advantage, and is ideal for annotating temporal and spatial expression profiles and the regulation mode of the ASS gene.
\end{abstract}

Keywords: Argininosuccinate synthetase, Transgenic mouse, Bacterial artificial chromosome, Green fluorescence protein, Developmental regulation, Tissue-specific regulation, Post-transcriptional regulation

\section{Background}

Argininosuccinate synthetase (ASS; EC 6.3.4.5) is an enzyme that functions in the catalysis of the conversion of citrulline and aspartate to argininosuccinate, which is further converted to arginine by argininosuccinate lyase $[1,2]$. ASS catalyzes the rate-limiting step in arginine biosynthesis. Arginine plays a role in the synthesis of

\footnotetext{
* Correspondence: su31659@gmail.com
'Institute of Microbiology \& Immunology, National Yang-Ming University, 112

'Institute of Microbiology \& Immunology, National Yang-Ming University, 112 Taipei, Taiwan 2Department of Medical Research, Taipei Veterans General Hospital, 112
Taipei, Taiwan 2Department
Taipei, Taiwan
}

\section{Biomed Central}

(c) 2014 Shiue et al.; licensee BioMed Central Ltd. This is an Open Access article distributed under the terms of the Creative Commons Attribution License (http://creativecommons.org/licenses/by/4.0), which permits unrestricted use, distribution, and reproduction in any medium, provided the original work is properly credited. The Creative Commons Public Domain Dedication waiver (http://creativecommons.org/publicdomain/zero/1.0/) applies to the data made available in this article unless otherwise stated. urea, nitric oxide $(\mathrm{NO})$, polyamines, proline, glutamate, creatine and agmatine [3]. Thus, ASS participates in fine-tuning production of $\mathrm{NO}$ and others to maintain cellular homeostasis in response to cellular and environmental stimuli. Conceivably, ASS, one of the key enzymes involving in arginine metabolism, is subjected to various mechanisms of regulation in both physiological and disease states.

Hormones, such as glucocorticoid, glucagon and insulin, are major regulators of the expression of urea cycle enzymes in the liver [1,2]. We have previously identified that the cAMP response element (CRE) located at about

ras otherwise stated. 
$10 \mathrm{~kb}$ upstream of the transcription start site of the human ASS gene is most likely the target site of the CREbinding protein (CREB) to mediate glucagon action [4]. However, the mechanism by which glucocorticoid and insulin act on ASS expression remains unknown. On the other hand, ASS expression in non-hepatic cells were shown to be induced by interleukin- $1 \beta$ through NF- $k B$ activation acting at a putative NF- $\mathrm{kB}$ binding site at the ASS promoter [5]. Moreover, the proximal promoter of the ASS gene was shown to contain an E-box recognized by c-Myc and HIF- $1 \alpha$, and a GC-box targeted by Sp4 where ASS expression involves interactions between the positive transcriptional factors $\mathrm{c}-\mathrm{Myc}$ and $\mathrm{Sp} 4$ and the negative factor HIF-1 $\alpha$ [6]. In addition to regulation at transcription initiation, we have also identified a novel post-transcriptional event affecting ASS nuclear precursor RNA stability in the canavanine-resistant variants of a human squamous cell carcinoma line, RPMI 2650 [7]. These variants express 200-fold increased levels of ASS mRNA as compared to the parental cells [8]. The canavanine-resistant variants have increased ASS activities, and are presumably resistant to canavanine because of increased conversion of citrulline to arginine. The post-transcriptional regulation identified in the canavanine-resistant variants may have physiological relevance since it has the advantage of being faster than transcriptional regulation. One would image that under particular circumstances such as inflammation, cells may employ such a mode of regulation to produce higher levels of ASS mRNA to meet the need for NO production. It is worth noting that similar canavanineresistant cells have been isolated from lymphoblasts [9].

To identify cellular targets or events that employ such regulation, a suitable ASS expression profiling system is essential. To this end, we took BAC (bacterial artificial chromosome) containing the entire human ASS structural gene of $57 \mathrm{~kb}$ flanked by $97 \mathrm{~kb}$ and $16 \mathrm{~kb}$ of genomic sequences at its $5^{\prime}$ - and $3^{\prime}$-ends, respectively, as a starting construct to knock-in the EGFP (enhanced green fluorescence protein) coding sequence. Two types of ASS-EGFP transgene were constructed (Figure 1). One is designated as a transcription reporter, $T g(A S S-E x 3-E G F P)$. In such a construct, $E G F P$ is knocked in at a position of authentic initiation codon residing in exon 3 of the ASS gene where EGFP transcription termination is regulated by a SV40 poly(A) signal. The EGFP level in this construct would, therefore, essentially reflect the transcription activities of the ASS gene. Another one is designated as a transcription/post-transcription couple reporter, $T g(A S S-E x 16-$ $E G F P)$, in which the EGFP knock-in sequence also contains the sequence of the internal ribosome entry site (IRES), and the hybrid sequence is inserted into exon 16, the terminal exon of the ASS gene, at site between the ASS stop codon and the polyA signal. In such a configuration, the human ASS and the EGFP genes are transcribed as a bicistronic RNA, and EGFP, the downstream cistron, is translated by the IRES mechanism. Thus, EGFP activity expressed from such a transgene is subjected to both transcriptional and post-transcriptional regulation as that of the endogenous ASS mRNA. Using transgenic mice carrying these ASS-EGFP reporters, we show here that location of EGFP in the liver and kidney, the major organ of ASS production, is essentially mimic that of the endogenous Ass gene, suggesting sufficient regulatory element(s) is included in the ASS-EGFP transgene.

To obtain the temporal and spatial expression profiles of ASS transgene, major efforts in this study are to establish the developmental expression pattern of ASS-EGFP in the liver, the organ for urea production, and in the intestine and kidney where arginine biosynthesis occurs. By comparing the expression patterns between $T g(A S S$ $E x 3-E G F P)$ that are carrying the transcription reporter and $\operatorname{Tg}(A S S-E x 16-E G F P)$ that are carrying the transcription/post-transcription couple reporter, we aim to deduce at which level the expression control acts during development. Moreover, the ASS transgene is of human origin. It would be of interest to know how a human gene behaved in the mouse genetic background.

\section{Methods}

\section{Animals}

Mice were housed in a specific pathogen-free (SPF) area of the animal room in the Taipei Veterans General Hospital and were maintained according to protocols approved by the Animal Care and Use Committee of Taipei Veterans General Hospital. The male transgenic mice of the $\mathrm{FVB} / \mathrm{N}$ strain carrying the ASS-EGFP transgene, i.e., $\operatorname{Tg}(A S S-E x 3-E G F P)$ or $T g(A S S-E x 16-E G F P)$, were mated with wild-type $\mathrm{FVB} / \mathrm{N}$ female where parturition occurred on day 17.5 or 18.5 after conception. Progenies carrying EGFP transgene were identified by visualization of fluorescence of two-week old littermates by a portable fluorescence detection system. The transgenic mouse lines $T g(A S S-E x 3-E G F P) T s u$ and $T g$ (ASS-Ex16-EGFP)Tsu have been deposited in National Laboratory Animal Center, Taiwan, and are available for researchers on requests.

\section{Histological study}

Histological study was performed following standard protocols [10]. In brief, mice were sacrificed by anesthetized with $\mathrm{CO}_{2}$ and tissue collected was fixed in $4 \%$ buffered paraformaldehyde. For frozen section, after dehydration in graded sucrose solution, tissue was embedded in OCT (Optimal Cutting Temperature) compound (Tissue Tec, Sakara, Torrance, CA). Serial sections were performed with Leica cryostat (Leica Biosystems, Wetzlar, Germany) and mounted onto slides to examine EGFP expression by 


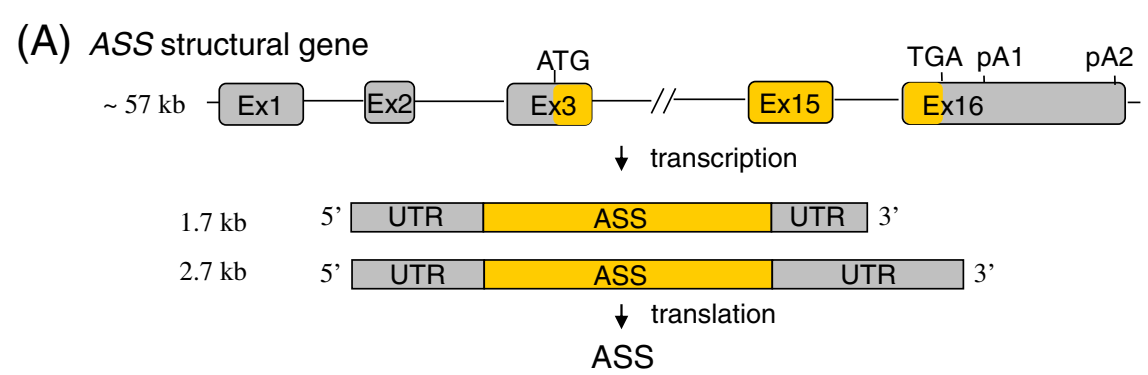

(B) $\operatorname{Tg}(A S S-E x 3-E G F P)$

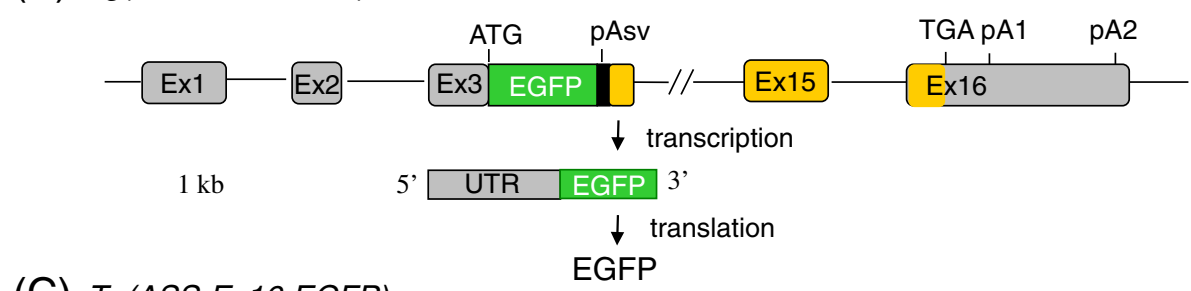

(C) $\operatorname{Tg}(A S S-E x 16-E G F P)$

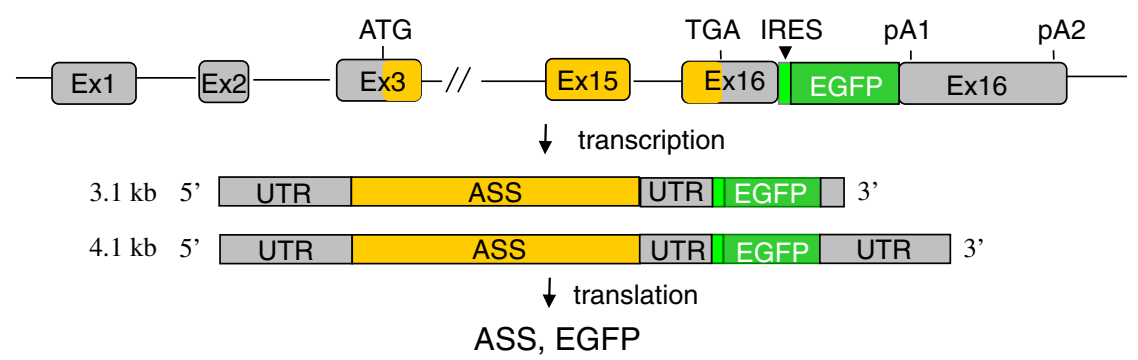

Figure 1 Schematic representation of the human ASS gene and the ASS-EGFP transgene. (A) Structure of the human ASS gene. Human ASS gene composes of 16 exons with the initiation codon (ATG) residing in exon 3 and the termination codon (TGA) in exon 16. There are two poly(A) signals, pA1 and pA2, residing in exon 16 to produce ASS mRNA of $1.7 \mathrm{~kb}$ and $2.7 \mathrm{~kb}$. UTR: untranslated region. (B) Structure of the Tg(ASS-Ex3EGFP) transgene. The EGFP coding sequence is inserted right after the initiation codon of the ASS gene. Transcription initiated from the ASS promoter is terminated by a SV40 poly(A) signal, pAsv, residing immediate downstream of the EGFP gene. EGFP but not ASS protein is produced from this transgene. (C) Structure of the Tg(ASS-Ex16-EGFP) transgene. EGFP is inserted in exon 16 between the termination codon and pA1 of the ASS gene. Depending on which poly(A) signal is used, bicistronic mRNAs of $3.1 \mathrm{~kb}$ and $4.1 \mathrm{~kb}$ are produced. A sequence of IRES (internal ribosome entrance site) is inserted in front of EGFP to facilitate EGFP protein translation. Both ASS and EGFP proteins are produced from this transgene.

a fluorescence microscope. Slides were counterstained with DAPI (4',6-diamidino-2-phenylindole) (Roche Applied Science, Indianapolis, IN). For immunohistochemical studies, tissues fixed in buffered paraformaldehyde and embedded in paraffin were deparaffinized, hydrated in graded ethanol, and heated in $10 \mathrm{mM}$ sodium citrate ( $\mathrm{pH}$ 6.0) plus $0.1 \% \mathrm{NP}-40$ by microwave oven to retrieve the antigens. The remaining activity of endogenous peroxidase activity was quenched with hydrogen peroxide. After blocking, the slides were incubated with a primary antibody of GFP (1:100 dilution, anti-GFP, rabbit polyclonal antibody) (Chemicon, Billerica, MA) or 1:100 dilution of a mouse monoclonal anti-ASS antibody (BD Biosciences, San Jose, CA) overnight at $4^{\circ} \mathrm{C}$. Subsequently, the slides were incubated with the biotinylated secondary antibody and streptavidin conjugatedHRP (horseradish peroxidase). The HRP was then visualized by the application of substrate chromogen DAB (diaminobenzidine) (Dako, Glostrup, Denmark) to give brown color where the slides were counterstained with hematoxylin.

\section{RNA isolation and S1 nuclease mapping analysis}

For total RNA isolation, tissue was first grinded to powder cooled in liquid nitrogen. The frozen powder was transferred to a MagNA Lyser tube (Roche Applied Science, Indianapolis, IN) filled with $1 \mathrm{ml}$ TRIzol (Invitrogen, Carlsbad, CA) and homogenized immediately by MagNA Lyser homogenizer (Roche Applied Science, Indianapolis, IN). The supernatant was used in RNA isolation following manufacturer's instructions. For S1 nuclease mapping, appropriate restriction enzymedigested DNA fragment was labeled at the 5 '-end with $\left[\gamma^{-}{ }^{32} \mathrm{P}\right]$ ATP and T4 polynucleotide kinase [11]. The labeled DNA probe was hybridized to total RNAs prepared from the mouse tissues. The DNA-RNA hybrids that resisted to $\mathrm{S} 1$ nuclease digestion were electrophoresed through a $4 \%$ polyacrylamide gel containing $7 \mathrm{M}$ 
urea. Gel was dried and analyzed either by autoradiography or by a phosphorimager (Molecular Dynamics, Sunnyvale, CA).

\section{Results and discussion}

ASS-EGFP expression during liver development

ASS expression in the liver has been shown to be developmentally regulated. In the rat, Ass mRNA is first detected in E15.5 fetal stage and increases continuously to neonatal stage [12]. To visualize ASS expression during liver development and to study whether post-transcriptional regulation is involved, studies were taken using transgenic mice $T g$ (ASS-Ex3-EGFP) 3GTsu and Tg(ASS-Ex3-EGFP) 3JTsu, abbreviated as $3 \mathrm{G}$ and 3J, that carry, respectively, 30 and 2 copies of the transcription reporter and $T g(A S S-E x 16-$ EGFP) 16ETsu and Tg(ASS-Ex16-EGFP) 16FTsu, abbreviated as $16 \mathrm{E}$ and $16 \mathrm{~F}$, that carry, respectively, 10 and 5 copies of the transcription/post-transcription couple reporter. EGFP fluorescence of liver tissues taken from fetuses at E14.5 to E17.5 (some to E18.5), 1 to 7 days and 2, 3 and 4 weeks after birth were examined directly under a fluorescence dissecting microscope (Figure 2A). Images were captured with 1.25-fold magnification for samples at stages E14.5 to D7 and 0.7-fold for those at stages W1 to W4. Images D7 and W1 in Figure 2A were taken from an identical sample. The difference in their fluorescence brightness is mainly due to the fact that the image brightness is inversely proportional to the square of the transverse magnification [13]. Moreover, image was captured using a constant 4-second exposure time except in 3G line which was reduced to 1 second due to strong EGFP signals. At E14.5 stage, EGFP fluorescence was weak except in the 3G line (Figure 2A, bottom panel, exposed for 4 seconds). Considerable enhancement of EGFP signal could be seen at day 1 and increased gradually to 4 weeks. Similar patterns of expression could also be seen in the $16 \mathrm{E}$ and $16 \mathrm{~F}$ lines, except that the signals were weaker (Figure 2A). Variations in EGFP fluorescence among mouse littermates were also observed. For example, the EGFP fluorescence was relatively strong in a liver sample of $16 \mathrm{~F}$ (Figure 2A, $16 \mathrm{~F} \mathrm{D2).}$

Zonation of metabolic pathways is believed to be a mechanism leading to efficient use of precursor pools and energy in an organism [14]. In the rodent, urea cycle enzymes in the liver show zonation distribution, i.e., they are present predominantly in the periportal hepatocytes and declining gradually toward the pericentral hepatocytes. Such a distribution pattern occurs not only at the protein level but also in the mRNAs of these enzymes [14]. To study whether zonation can be visualized by EGFP fluorescence, frozen sections of the liver were examined. Similar EGFP zonation pattern could clearly be seen between transgenic lines carrying the transcription reporter, i.e., $3 G$ and 3J, and the transcription/post- transcription couple reporter, i.e., 16E and $16 \mathrm{~F}$ (Figure 2B). In the rodent, Ass RNA zonation has been shown to appear 2 days before birth [14]. In agreement, zonation gradually became apparent at E17.5 and E18.5 (Figure 2C, 3J line) where near-homogeneous EGFP fluorescence distribution was found at E14.5 and E15.5 stages (Figure 2C, 3G line). However, human ASS has been reported to be present in all hepatocytes with no marked zonation [15]. The ASS-EGFP transgene in this study is of human origin, and yet EGFP expression manifested zonation pattern similar to that of the rodent Ass. Apparently, establishment of zonation pattern is made at transcription initiation and such regulation may be influenced by cellular environment. Thus, human ASS gene when in the rodent background follows the expression pattern of the rodent.

\section{ASS-EGFP expression during kidney development}

Kidney is the major organ of arginine production in adult where ASS is the rate-limiting step in the conversion of citrulline to arginine [1]. Similar to the liver, EGFP fluorescence in the kidney was weak at E14.5 stage except that of the 3G line (Figure 3A, bottom panel). Marked enhancement of EGFP expression was observed during neonatal (Figure 3A, D1 stage). The ASS protein and mRNA have been shown to localize exclusively in the proximal tubules of renal cortex [16]. Indeed, in our transgenic mice, EGFP fluorescence was mainly found in the renal cortex area (Figure 3B). Renal cortex can be divided into alternate regions of cortical labyrinth and the medullary rays [17]. EGFP signal appeared in cortical labyrinth (Figure 3C, CL) and not in the medullary rays (Figure $3 \mathrm{C}, \mathrm{MR}$ ). The cortical labyrinth consists of glomeruli and convoluted tubules where EGFP signal appeared in the convoluted tubules and not in the glomeruli (Figure 3C, lower panel). To examine the kidney structure in detail, immunohistochemical studies using EGFP and ASS antibodies were performed (Figure 3D). Signals were found in the proximal convoluted tubules and the parietal epithelium but were absent in the glomeruli and the distal convoluted tubules when analyzed by either EGFP or ASS antibodies. Therefore, the ASS-EGFP transgene is faithfully expressed as an authentic ASS gene. On the other hand, to study the EGFP expression pattern during kidney development, kidney frozen sections in various developmental stages were examined (Figure 3E). At E14.5 and E15.5 stages, EGFP fluorescence was found to distribute rather homogenously in the entire kidney (Figure 3E, 3G line). Fluorescence in the structure of the renal pelvis was also visible. The nephrogenic zone can be divided into the cortex and medulla in mouse kidney at E15.5 [18]. During perinatal stage, EGFP fluorescence was found primarily in the cortex although weak signals were visible in the collecting 


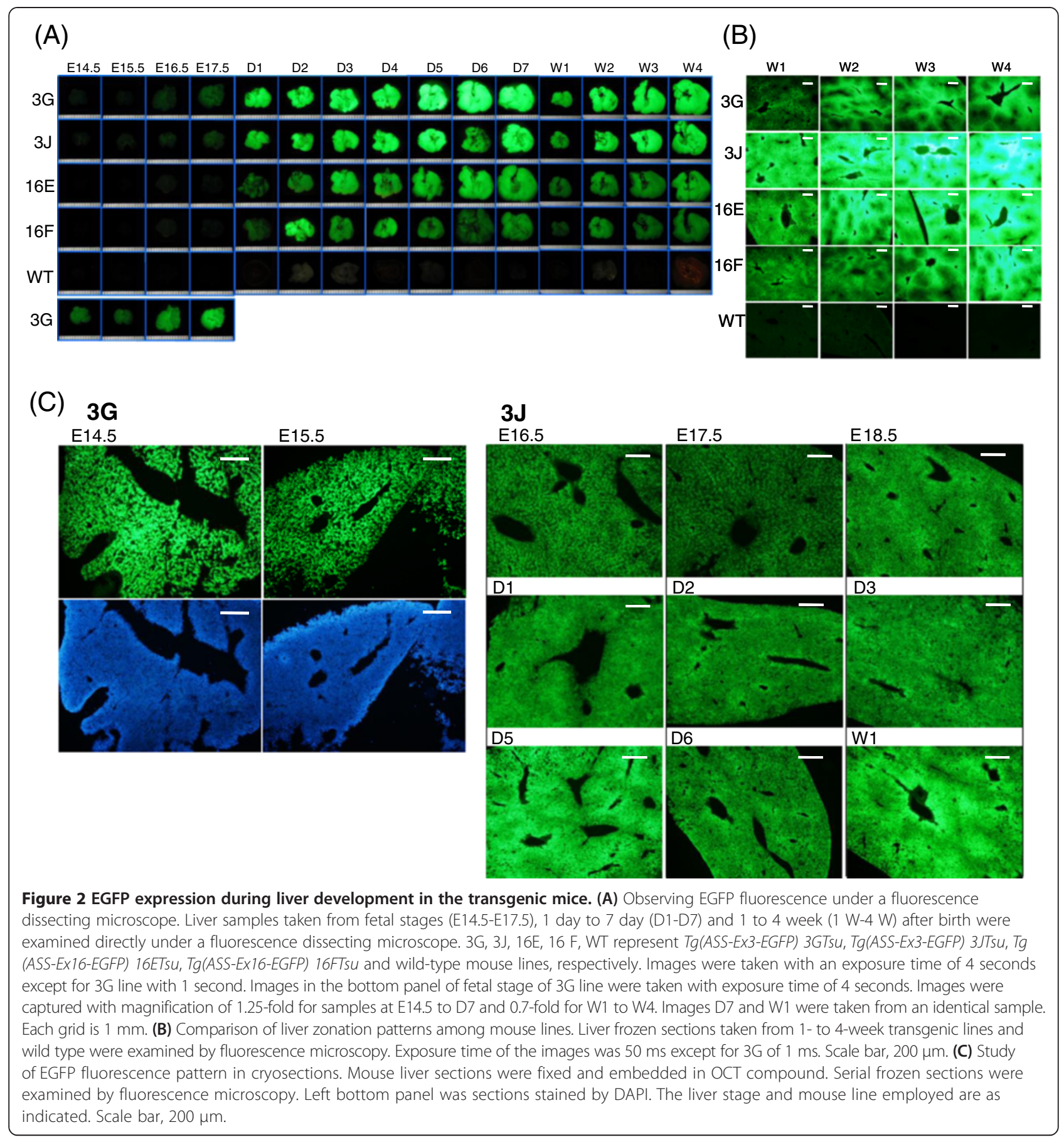

duct of the medulla (Figure 3E, stages E16.5-D2). At later stages, fluorescence was essentially seen in the cortex where areas of fluorescence increased. Thus, ASS-EGFP expression recaptures the feature of Ass gene in the mouse kidney [16].

\section{ASS-EGFP expression during intestine development}

The small intestine is the major organ in arginine synthesis during the perinatal stage of development, but gradually shifts to citrulline production. Citrulline generated is then taken up by the kidney where it is converted to arginine. The collaboration between the intestine and the kidney in arginine synthesis becomes established postnatally, constituting the intestinal-renal axis [19]. To study whether EGFP fluorescence in the small intestine in the ASS-EGFP transgenic mice can reproduce such regulation, EGFP fluorescence in the digestion system was examined directly under a fluorescence dissecting 


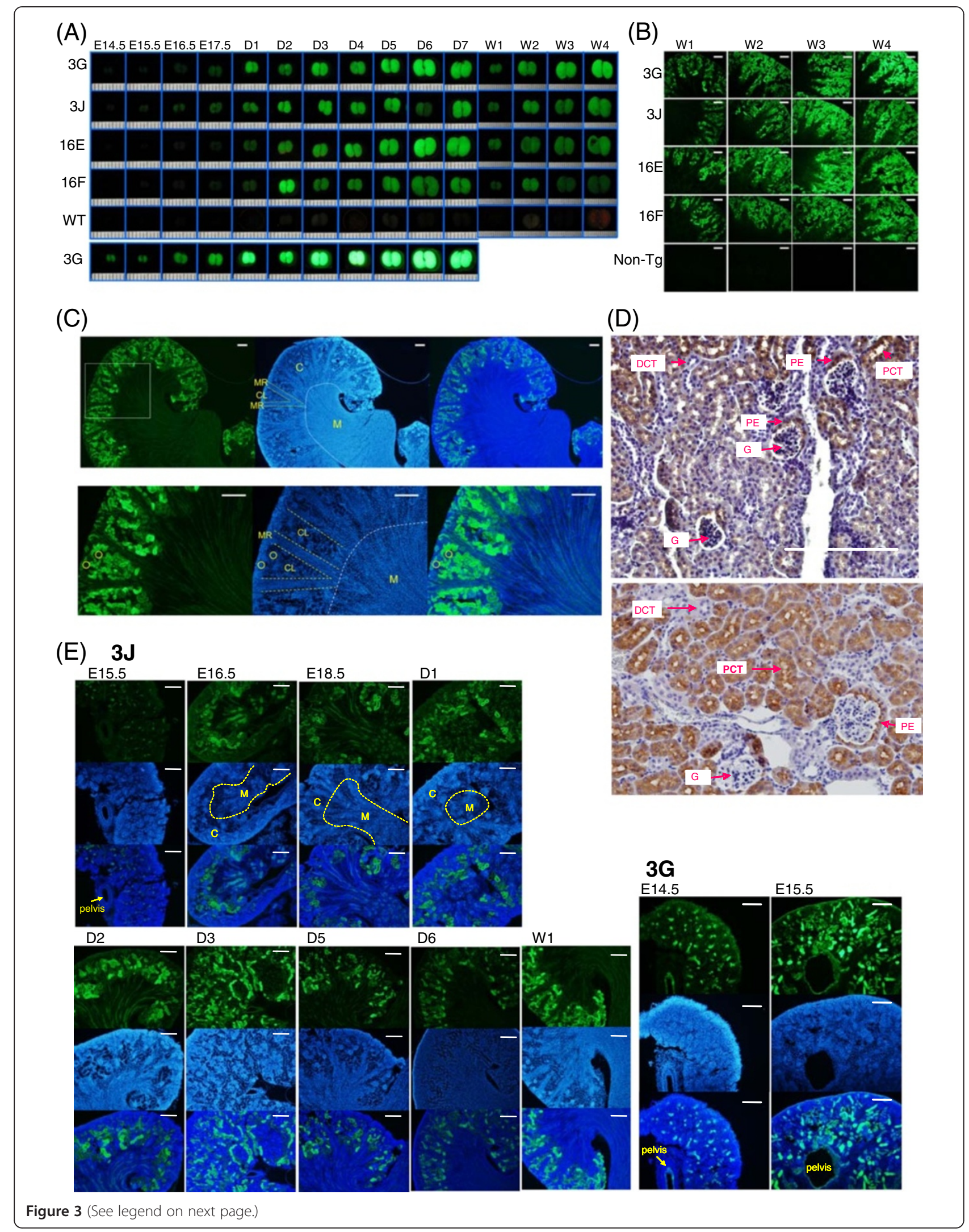


(See figure on previous page.)

Figure 3 EGFP expression during kidney development in the transgenic mice. (A) Study of EGFP fluorescence by fluorescence dissecting microscopy. Conditions were as described in the legends of Figure 2A. (B) Comparison of fluorescence patterns of the kidney among the mouse lines. Kidney frozen sections taken from 1- to 4-week transgenic mice and non-Tg controls were examined by fluorescence microscopy. Exposure time was $50 \mathrm{~ms}$ except for $3 \mathrm{G}$ of $1 \mathrm{~ms}$. Scale bar, 200 mm. (C) Localization of EGFP fluorescence. Frozen sections of kidneys from 1-week old transgenic mouse $3 \mathrm{~J}$ were examined by fluorescence microscopy. A region of enlarged image was selected (white box) and shown in the lower panel. In each panel: left, fluorescence image; middle, DAPI staining; right, fluorescence image merged with DAPI staining. Particular regions are distinguished by dotted lines: C: cortex; M: medulla; CL: cortical labyrinth; MR: medullary ray. The glomerulus is circled in yellow. (D) Immunohistochemical localization of EGFP and ASS. Paraffin sections of the kidney from 4 week-old transgenic mouse $3 G$ were incubated with an EGFP antibody (upper panel) or ASS antibody (lower panel). The slides were incubated with the biotinylated secondary antibody coupled with streptavidin conjugated-HRP and was visualized by the application of chromogen DAB to produce brown color when the slides were counterstained with hematoxylin. PCT: proximal convoluted tubule; DCT: distal convoluted tubule; G: glomerulus; PE: parietal epithelium. Scale bar, 200 mm. (E) EGFP fluorescence patterns during kidney development of the transgenic mice. Mouse kidney was fixed and embedded in OCT compound. Serial frozen sections were examined by fluorescence microscopy. In each panel: top, EGFP fluorescence image, middle, DAPI staining, bottom, EGFP fluorescence image merged with DAPI staining. The developmental stages and mouse lines employed are as indicated. Areas of the cortex $(\mathrm{C})$ and the medulla $(\mathrm{M})$ are distinguished by dotted lines. Scale bar, $200 \mu \mathrm{m}$.

microscope (Figure 4A). In contrast to the liver and kidney, the EGFP signals in the intestine were clearly visible in the fetal stages (Figure 4A). The signals persisted in to the perinatal stage but decreased gradually around the time of weaning (Figure 4A). It is noted that substantial auto-fluorescence could be seen in organs of a wild-type mouse, especially in the stomach (Figure 4A, WT). Such signals in part originated from the food intake (data not shown). On the other hand, De Jonge et al. [20] have studied temporal expression of urea cycle enzymes in the rat small intestine during perinatal development. By northern blot analysis and in situ hybridization, they found in the fetal stage that the proximal loops of the intestine expressed the Ass mRNAs at higher concentrations than the more distal loops. In agreement, frozen sections of the transgenic fetuses at E14.5 or E15.5 showed pronounced EGFP signals in the proximal loops of the intestine in the region closer to the liver (Figure 4B, white arrow) while only weak signals were seen in the distal parts of the intestine (Figure 4B, red arrow). In addition, EGFP signals were clearly seen in the enteric neurons of the myenteric ganglion (Figure 4B, MG). Thus, EGFP signals in the small intestine of the ASS-EGFP transgenic mice appear to recapture the temporospatial expression of rodent Ass.

\section{Expression profiling of EGFP and Ass mRNAs in the liver, kidney and intestine by $\mathrm{S} 1$ nuclease mapping}

Studies described above show that EGFP fluorescence in the liver, kidney and intestine examined by fluorescence microscopy either directly or after cryosectioning is useful to study ASS expression. In such analyses, no gross disparity in EGFP expression was found between mouse lines of $T g$ (ASS-Ex3-EGFP) Tsu and Tg(ASS-Ex16-EGFP) $T s u$. To be quantitative, EGFP mRNA profiles of the liver, kidney and intestine were examined by $\mathrm{S} 1$ nuclease mapping. In addition, the ASS-EGFP transgene is of human origin. To examine whether species-specific expression pattern existed, comparison was also made between expression profiles of the EGFP mRNA and the endogenous mouse Ass mRNA. Abundances of EGFP and Ass mRNAs from the liver, kidney and intestine in different developmental stages were analyzed and quantified by $\mathrm{S} 1$ nuclease mapping; where levels of EGFP and Ass mRNAs were first normalized to the mRNA level of Gapdh, a house keeping gene glyceraldehyde-3-phosphate dehydrogenase. The mRNA structures of EGFP, Ass and Gapdh, their 5 '-end labeled cDNA probes and the expected sizes of protected fragment are depicted in Figure 5A. The probes are designed such that the size of protected fragment by respective probes could easily be distinguished by denatured polyacrylamide gel electrophoresis. RNAs were hybridized in reaction containing individually end-labeled cDNA probes of EGFP, mouse Ass and mouse Gapdh. DNA-RNA duplexes were analyzed by electrophoresis after removing unhybridized RNA and single-stranded probes by $\mathrm{S} 1$ nuclease. It is worth noting that in this analysis, the human ASS mRNA expressed from the $\operatorname{Tg}(A S S-E x 16-$ EGFP) Tsu line cannot be detected by the mouse Ass probe. Moreover, to study RNA expression of a particular organ, same batch of the labeled probes was used to avoid signal variations due to differences in probe specific activities. Representative images acquired by the phosphorimager on the analysis of liver, kidney and intestinal RNAs and the expression profiles of EGFP and Ass mRNAs are presented (Figures 5, 6 and 7).

Analysis of liver RNA from fetal E14.5 stage to 4 week after birth, EGFP mRNA level was found to increase progressively during the development (Figure 5B). Significant variations in EGFP mRNA abundances among mouse littermates were found. Such variations were also noted when EGFP fluorescence of a particular organ was examined directly (Figures 2A, 3, 4A). To study whether EGFP expression profile differs between $T g(A S S-E x 3-$ EGFP) $T s u$ and $T g$ (ASS-Ex16-EGFP) Tsu lines during liver development, relative abundances of EGFP mRNAs was determined (Figure 5C, upper panel). The EGFP mRNA levels among the transgenic lines were found to be 


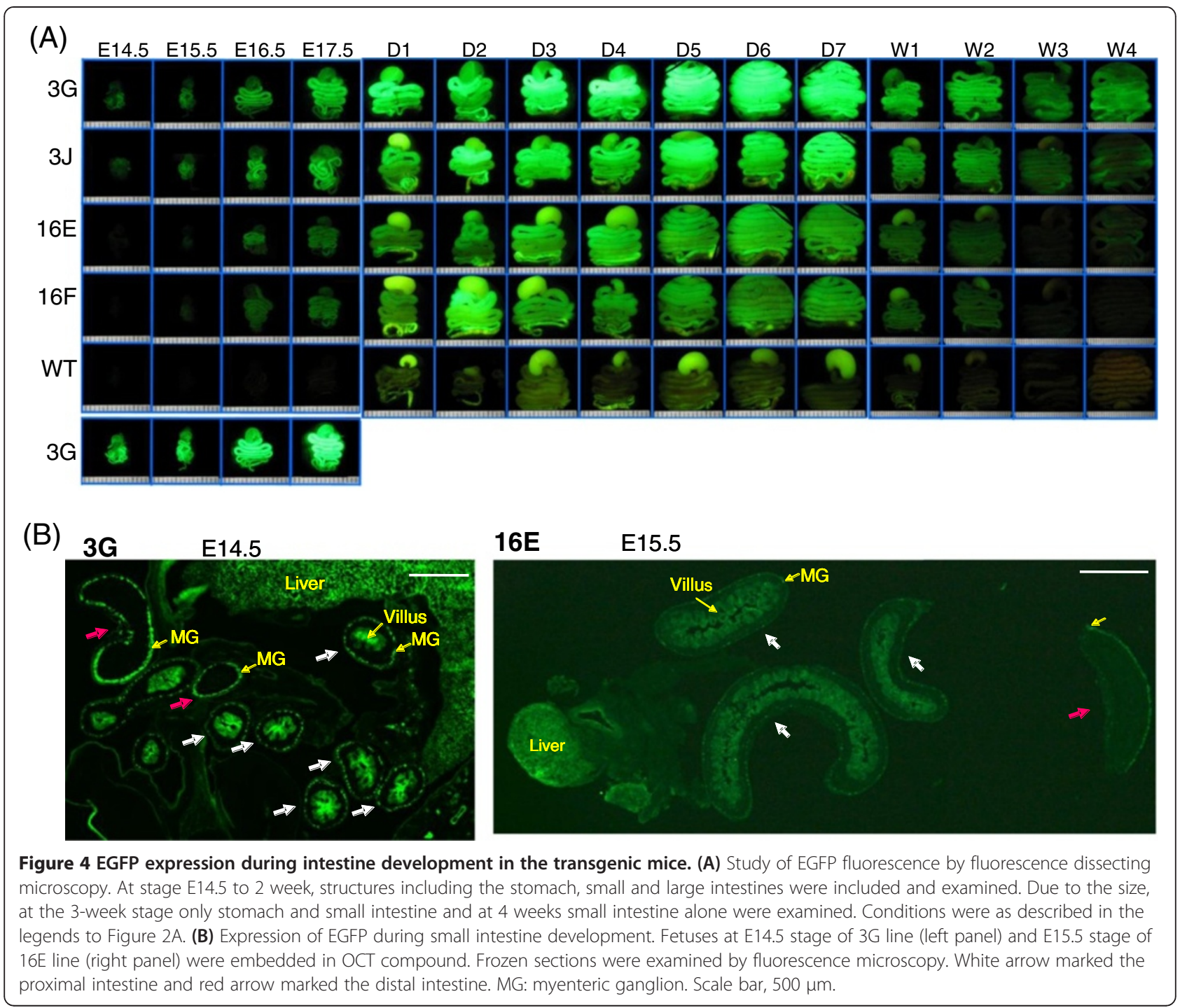

approximately reflecting their transgene copy numbers (Figure 5C, upper panel), suggesting that developmental ASS expression in the liver is mainly controlled at transcription initiation. If the novel post-transcriptional regulation affecting ASS nuclear precursor RNA stability similar to that in canavanine-resistant variants occurred in the $T g$ (ASS-Ex16-EGFP) Tsu line [7], one would expect the EGFP mRNA level in the $T g(A S S-E x 16-E G F P) T s u$ line to increase by one to two orders of magnitude from current levels. However, whether any subtle post-transcriptional regulation on ASS mRNA has taken place would require further studies. Nevertheless, both the 1.7- and 2.7-kb ASS mRNA species are highly stable with a half-life of approximately $15-20 \mathrm{~h}$ [21]. It is noted that among the organs examined, EGFP fluorescence in the $T g$ (ASS-Ex16-EGFP) Tsu line was generally weaker yet considerably high levels of EGFP mRNA could be detected. It is likely that EGFP in the $\operatorname{Tg}(A S S-E x 16-E G F P) T s u$ line, which is the downstream cistron, is translated by IRES mechanism which may be less efficient comparing to cap-dependent translation of EGFP in the $\operatorname{Tg}(A S S-E x 3-E G F P) T s u$ line.

In rodents, Ass gene expression, assayed by RNA or by activities, is reported to increase progressively towards birth reaching about $50 \%$ of adult liver value [1]. In contrast, in human fetuses, ASS activities reached $90 \%$ of the adult value at 36 weeks of gestation [22]. Our study showed that the EGFP mRNA abundances at birth were less than $40 \%$ of the adult values (Figure $5 \mathrm{C}$, upper panel). Thus, EGFP mRNA profile during liver development is similar to that of mouse Ass mRNA, suggesting that the human ASS gene in the mouse background follows the mouse Ass developmental pattern. Similar conclusion has been obtained as evidenced by liver zonation distribution of EGFP fluorescence (Figure 2B). Thus, the human ASS gene in the mouse genetic background follows mouse Ass developmental pattern. This is not 


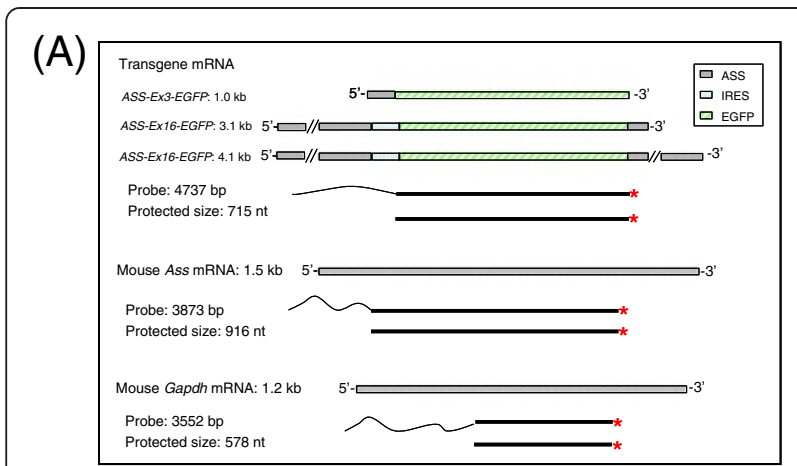

(B)

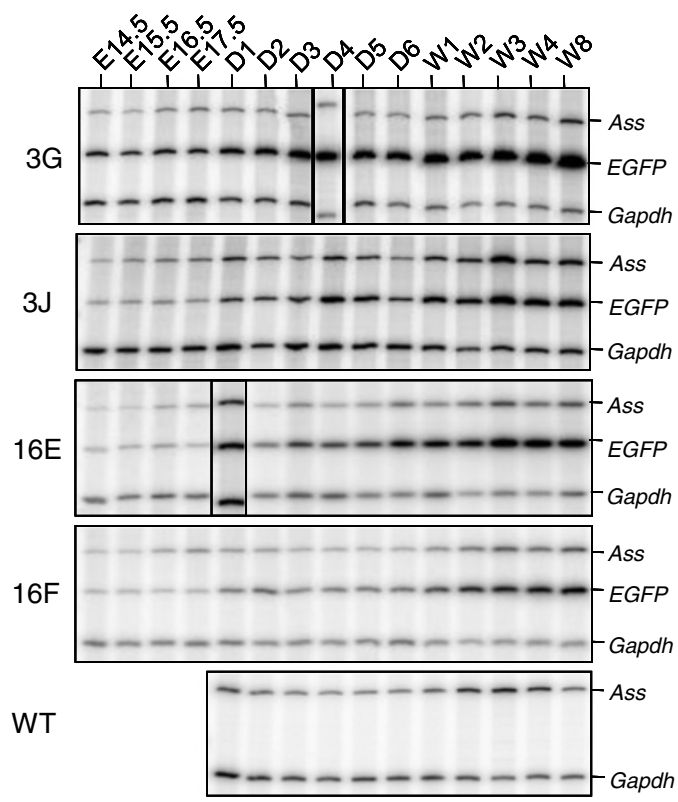

(C)

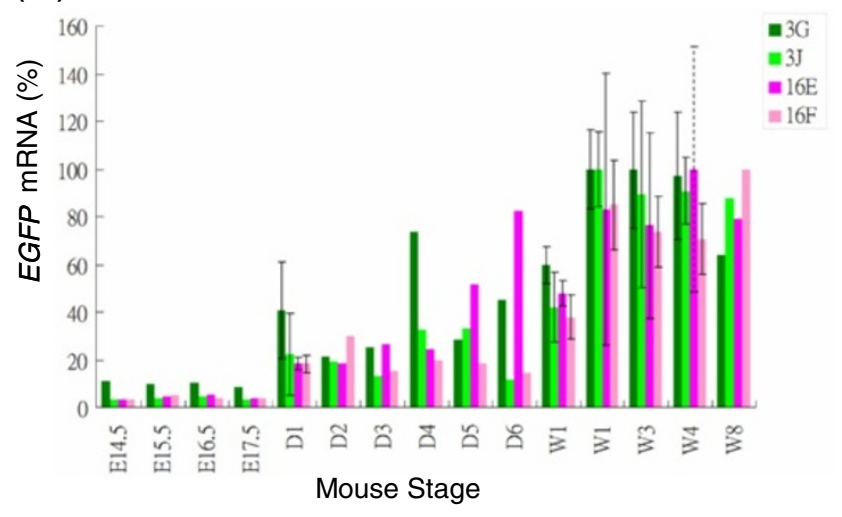

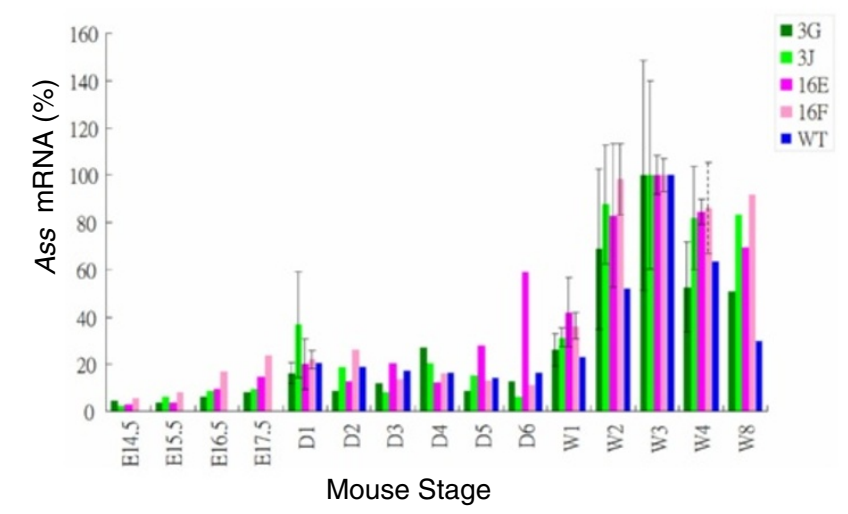

Figure 5 Determination of relative abundances of EGFP and mouse Ass mRNAs during liver development by S1 nuclease mapping.

(A) Strategy of S1 nuclease mapping. RNA structures, DNA probes and the predicted protected probe size after S1 nuclease digestion are depicted. Stars indicate ${ }^{32} \mathrm{P}$ 5'-end labels; wavy line at one end of the labeled probe indicates vector sequences that distinguish a re-annealed probe from a probe that was protected by hybridization to RNA. (B) Phosphorimage analysis of the protected fragments from S1 nuclease mapping. RNAs isolated from various stages of liver development (indicated on top) were hybridized to the $5^{\prime}$ end-labeled probes and the DNA-RNA hybrids that resisted S1 nuclease digestion were electrophoresed through a 4\% polyacrylamide gel containing $7 \mathrm{M}$ urea. Gel was dried and analyzed by a phosphorimager. The protected fragments corresponding to mRNAs of mouse Ass, EGFP transgene and mouse Gapdh are marked on the right. Mouse lines from which RNAs were obtained are indicated on the left. (C) EGFP and Ass mRNA profiles during liver development. The intensities of hybridization signals of EGFP, Ass and Gapdh mRNAs were quantified by a phosphorimager where the levels of EGFP and Ass mRNAs were normalized to the level of the Gapdh mRNA. Relative abundances of EGFP mRNA (upper panel) and Ass mRNA (lower panel) during liver development are plotted by taking the stage of the highest level as 100\%. Standard deviation (solid line) is shown if analysis was performed at least 3 times. If performed twice, average with interval of two values is shown (dotted line).

surprising since the ASS gene during liver development is known to subject to both hormonal and nutritional regulation [1,2]. Therefore, cellular environment plays important role in ASS expression. The results indicate that the cis-elements required for ASS expression during liver development are similar between the human and the mouse, and the ASS-EGFP transgene carries sufficient elements to execute such regulation.

To study whether the transgene expression may affect endogenous Ass gene expression, levels of Ass mRNA were compared between the $3 \mathrm{G}$ and $3 \mathrm{~J}$ lines of $\mathrm{Tg}(\mathrm{ASS}$ -
Ex3-EGFP) Tsu, which carry 30 and 2 copies of transgene, respectively. Indeed, a significantly higher level of EGFP mRNA was found in the $3 G$ line compared to that of the 3J line, yet comparable abundance of Ass mRNA was detected among the transgenic lines $3 \mathrm{G}$ and $3 \mathrm{~J}$ and that of the wild-type mouse (Figure 5C, lower panel). Similar results were found in the analysis of the Tg(ASS-Ex16-EGFP) Tsu line (Figure 5C, lower panel 16E and $16 \mathrm{~F}$ ). Therefore, even in the presence of high copies of the transgene, no apparent sequestration of transcription factors to affect endogenous gene expression occurs. 


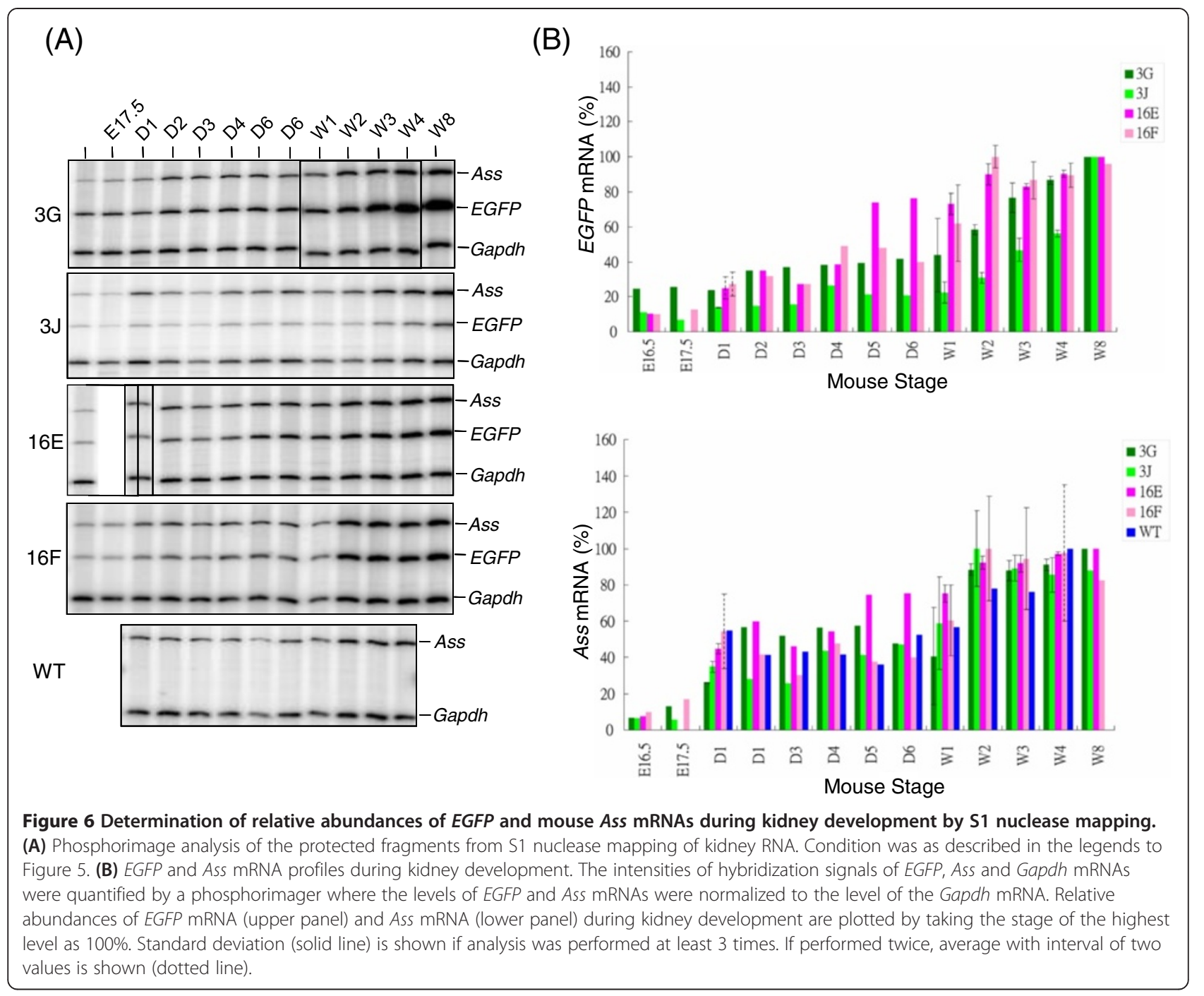

Approach similar to that of the liver was taken to study EGFP mRNA expression during kidney development (Figure 6). However, due to limit amounts of sample in fetal E14.5 and E15.5 stages, analysis started at fetus E16.5 stage. Representative images of RNA analysis by $\mathrm{S} 1$ nuclease mapping are shown in Figure 6A. The EGFP expression profile was similar between the $T g$ (ASS-Ex3-EGFP) Tsu and Tg(ASS-Ex16-EGFP) Tsu lines (Figure 6B, upper panel), suggesting ASS expression during kidney development is controlled mainly at the transcription level. By comparing Ass mRNA levels between wild-type and transgenic lines, one concludes that expression of the transgene does not interfere with expression of the endogenous Ass gene during kidney development in the mouse (Figure 6B, lower panel).

To study EGFP mRNA expression profiles in the intestine, RNA of the digestion system including stomach to rectum at the fetal stage was isolated. After birth, only RNA from the small intestine was analyzed. Because
EGFP fluorescence intensity was found to vary among different sections of the small intestine (data not shown), to avoid complication, the entire small intestine was collected and used for RNA isolation. In this study, we again showed that EGFP mRNA abundance during intestine development is mainly controlled at the transcription initiation and expression of the transgene did not interfere with endogenous Ass expression (Figure 7). Interestingly, although there were similarities between the expression profiles of EGFP and Ass mRNAs, some differences did exist (Figure 7B). For example, in contrast to EGFP mRNA which showed smooth increases in its abundance perinatally, a rather sharp increase in Ass mRNA abundance at E17.5 stage was observed (Figure 7B). Moreover, the Ass mRNA levels declined sharply at 3 weeks of age, but substantial EGFP mRNA could be detected at this stage in both $T g(A S S-E x 3-E G F P) T s u$ or $T g$ (ASS-Ex16-EGFP) Tsu lines (Figure 7B). In this regard, a previous study of arginine-metabolizing enzymes in the 


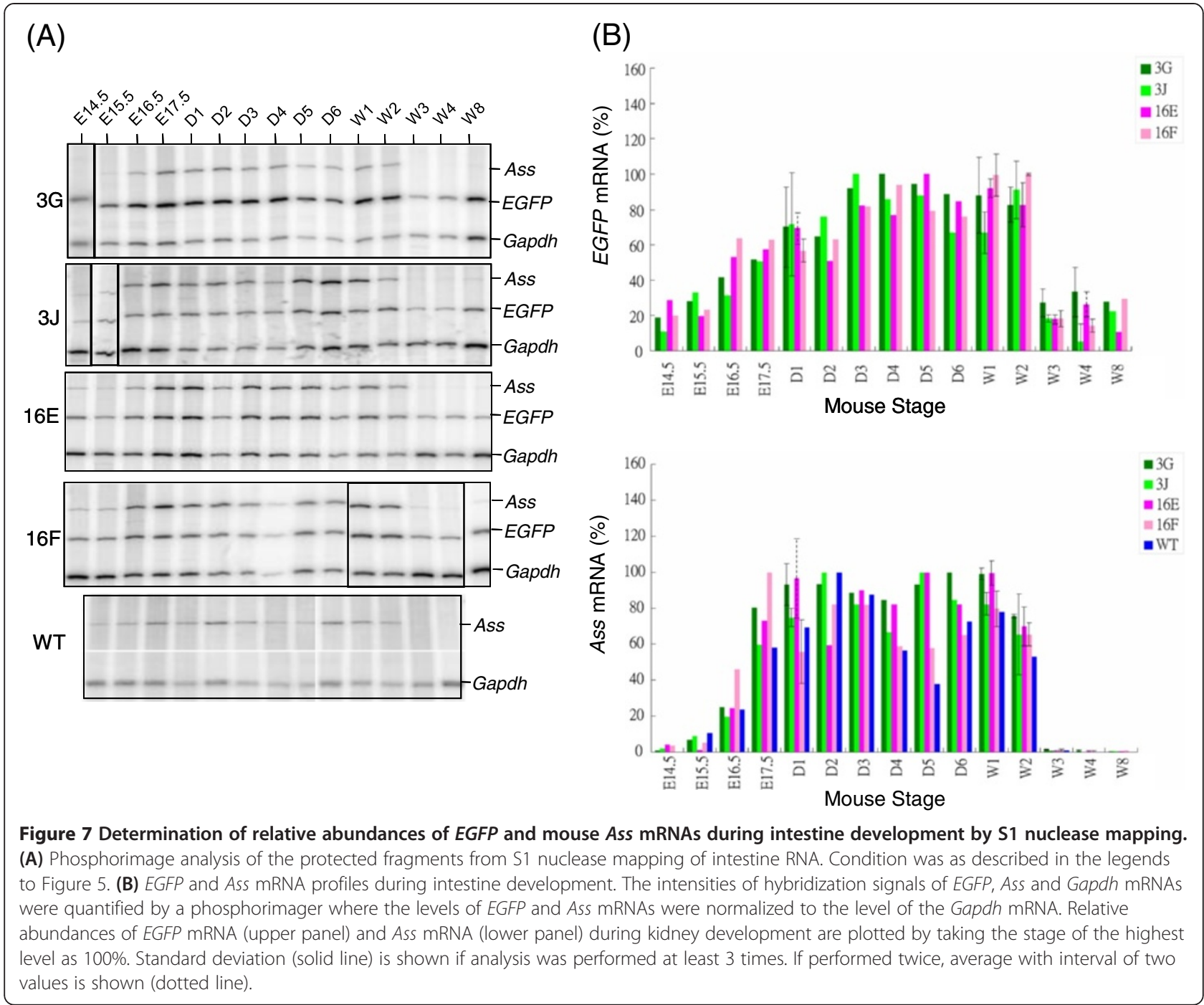

developing rat small intestine found that mRNA levels of all genes in arginine metabolism were highest during the suckling period where Ass mRNAs declined to hardly detectable levels in the second postnatal week [20]. On the other hand, in piglets, net synthesis of arginine declines more gradually in the small intestine and is still present at 7 weeks of age [23]. The perinatal human intestine resembles that of piglets in that ASS activities are highest during the suckling period and declines to low levels around weaning and then rises again [24]. Thus, in contrast to mouse Ass mRNA that disappears completely at 3 week, the EGFP mRNA expression profile of the transgenic mice resembles human ASS in that substantial levels of EGFP mRNA could still be detected at 8 weeks of age (Figure 7). Apparently, ASS gene in humans and pigs has specific ciselement(s) which differ from those of the rodent in regulating ASS expression in the small intestine. By comparison of the upstream ASS gene sequences in human, pig and mouse, such cis-element(s) may be deduced.
Using transgenic mouse system, we show in this study that developmental- and tissue-specific ASS expression of liver, kidney and intestine is mainly controlled at transcription initiation. This is not surprising since transcription initiation, the first step of gene regulation, is the most important mechanism to determine whether or not genes are expressed and then, how much of encoded mRNAs are produced. On the other hand, the novel post-transcriptional regulation identified in canavanineresistant variants may be so genetically programmed in particular cell types to meet immediately the demand for high ASS activity. Our preliminary study indicates such regulation may occur during mouse fetal brain development ( $\mathrm{T} \mathrm{Su}$, unpublished data). The region of the brain involved is currently under characterization. On the other hand, the ASS gene in this transgenic mouse system is of human origin. How would the transcriptional programs of human gene follow in mouse genetic background? In this regard, Wilson et al., [25] have initiated 
an important study by taken a mouse model of Down syndrome in which mouse cells contain a copy of human chromosome 21. They concluded that genetic sequence is largely responsible for directing transcriptional programs in homologous tissues. Others such as interspecies differences in epigenetic machinery, cellular environment, and transcription factors themselves play secondary roles. Our study shows that developmental program of ASS-EGFP transgene in liver is similar to rodent, suggesting genetic elements determining liver development are comparable between human and rodent. Thus, cellular environments play important role in shaping ASS-EGFP transcriptional program during liver development. On the other hand, the time course of EGFP expression in small intestine resembles that of human ASS gene, suggesting the presence of particular genetic sequence(s) in ASS gene that dictates human intestine development.

\section{Conclusions}

We demonstrate that this transgenic mouse system is ideal for annotation of temporal and spatial expression profiles of the ASS gene. In particular, the $T g(A S S-E x 3-$ EGFP) 3GTsu line, containing 30 copies of the transgene, generates strong EGFP signals, and is, thus, useful in revealing weak expression. It is conceivable that a comprehensive knowledge of cell types expressing ASS may provide insights into the function. Such knowledge should facilitate investigation of the role of ASS in conditions of physiological and disease states, especially when ASS mRNA or protein are found not to be expressed in many tumours including hepatocellular carcinoma (HCC), melanoma, some mesotheliomas, renal cell cancers, sarcomas and lymphomas [26]. As a result, arginine deprivation employing the pegylated form of arginine deiminase (ADI-PEG20) as a targeted therapy is currently in clinical trials for patients with HCC and melanoma [26]. In this respect, feature of down-regulated expression of ASS in HCC has been recaptured in our ASS-EGFP transgenic mouse model (manuscript in preparation). Thus, questions such as physiological or pathophysiological response of ASS expression stimulated by a variety of signals may be tackled using this system.

\section{Competing interests}

The authors declare that they have no competing interests.

\section{Authors' contributions}

SS carried out the experiments. MH participated in mouse study. TS conceived of the study, participated in its design and writing the manuscript. All authors read and approved the final manuscript.

\section{Acknowledgements}

We thank Kong-Bung Choo for critical reading and editing of the manuscript Chun-Ming Chen, Ting-Fen Tsai and Hsian-guey Hsieh for technical support. This work was supported by grants NSC 99-2320-B-075-001 from the National Science Council and by grants V98C1-188 and V100C-107 from the Taipei Veterans General Hospital, Taiwan.
Received: 11 March 2014 Accepted: 21 April 2014

Published: 13 May 2014

\section{References}

1. Husson A, Brasse-Lagnel C, Fairand A, Renouf S, Lavoinne A: Argininosuccinate synthetase from the urea cycle to the citrulline-NO cycle. Eur J Biochem 2003, 270:1887-1899

2. Haines RJ, Pendleton LC, Eichler DC: Argininosuccinate synthase: at the center of arginine metabolism. Int J Biochem Mol Biol 2011, 2:8-23.

3. Morris SM Jr: Arginine: beyond protein. Am J Clin Nutr 2006, 83:508S-512S.

4. Guei TR, Liu MC, Yang CP, Su TS: Liver-specific cAMP responsive element in the human argininosuccinate synthetase gene. Biochem Biophys Res Commun 2008, 377:257-261.

5. Brasse-Lagnel C, Lavoinne A, Loeber D, Fairand A, Bôle-Feysot C, Deniel N, Husson A: Glutamine and interleukin-1 $\beta$ interact at the level of Sp1 and nuclear factor- $\mathrm{kB}$ to regulate argininosuccinate synthetase gene expression. FEBS J 2007, 274:5250-5262.

6. Tsai WB, Aiba I, Lee SY, Feun L, Savaraj N, Kuo MT: Resistance to arginine deiminase treatment in melanoma cells is associated with induced argininosuccinate synthetase expression involving c-Myc/HIF-1a/Sp4. Mol Cancer Ther 2009, 8:3223-3233.

7. Tsai TF, Su TS: A nuclear post-transcriptional event responsible for overproduction of argininosuccinate synthetase in a canavanine-resistant variant of a human epithelial cell line. Eur J Biochem 1995, 229:233-238.

8. Su TS, Beaudet AL, O'Brien WE: Increased translatable messenger ribonucleic acid for argininosuccinate synthetase in canavanine-resistant human cells. Biochemistry 1981, 20:2956-2960.

9. Jacoby LB: Canavanine-resistant variants of human lymphoblasts. Somat Cell Genet 1978, 4:221-231.

10. Larsson LI: Immunocytochemistry: Theory and Practice. Boca Raton, Florida: CRC Press Inc; 1988.

11. Sambrook J, Russell DW: Molecular Cloning: A Laboratory Manual. 3rd edition. Cold Spring Harbor Laboratory Press: Cold Spring Harbor; 2001

12. Bourgeois P, Harlin JC, Renouf S, Goutal I, Fairand A, Husson A: Regulation of argininosuccinate synthetase mRNA level in rat foetal hepatocytes. Eur J Biochem 1997, 249:669-674.

13. Hutmacher DW, Dokland T, Ng MML: Techniques in Microscopy for Biomedical Applications. 1st edition. Singapore: World Scientific Pub Co Inc; 2006.

14. Dingemanse MA, De Jonge WJ, De Boer PAJ, Mori M, Lamers WH, Moorman AF: Development of the ornithine cycle in rat liver: Zonation of a metabolic pathway. Hepatology 1996, 24:407-411.

15. Saheki T, Yagi Y, Sase M, Nakano K, Sato E: Immunohistochemical localization of argininosuccinate synthetase in the liver of control and citrullinemic patients. Biomed Res Tokyo 1983, 4:235-238.

16. Morris SM Jr, Sweeney WE Jr, Kepka DM, O'Brien WE, Avner ED: Localization of arginine biosynthetic enzymes in renal proximal tubules and abundance of mRNA during development. Pediatr Res 1991, 29:151-154

17. Kriz W, Koepsell H: The structural organization of the mouse kidney. Anat Embryol 1974, 144:137-163.

18. Georgas K, Rumballe B, Wilkinson L, Chiu HS, Lesieur E, Gilbert T, Little MH: Use of dual section mRNA in situ hybridisation/immunohistochemistry to clarify gene expression patterns during the early stages of nephron development in the embryo and in the mature nephron of the adult mouse kidney. Histochem Cell Biol 2008, 130:927-942.

19. Morris SM Jr: Regulation of enzymes of the urea cycle and arginine metabolism. Annu Rev Nutr 2002, 22:87-105.

20. De Jonge WJ, Dingemanse MA, De Boer PAJ, Lamers WH, Moorman AFM: Arginine-metabolizing enzymes in the developing rat small intestine. Pediatr Res 1998, 43:442-451.

21. Tsai TF: Regulation of Human Argininosuccinate Synthetase Gene Expression. PhD Thesis. Taiwan: National Yang-Ming University, Institute of Microbiology \& Immunology; 1995.

22. Ali Baig MM, Habibullah CM, Swamy M, Hassan SI, Zaman TU, Ayesha Q, Devi BG: Studies on urea cycle enzyme levels in the human fetal liver at different gestational ages. Pediatr Res 1992, 31:143-145.

23. Stoll B, Henry J, Reeds PJ, Yu H, Jahoor F, Burrin DG: Catabolism dominates the first-pass intestinal metabolism of dietary essential amino acids in milk protein-fed piglets. J Nutr 1998, 128:606-614.

24. Köhler ES, Sankaranarayanan S, van Ginneken CJ, van Dijk P, Vermeulen JL, Ruijter JM, Lamers WH, Bruder E: The human neonatal small intestine has 
the potential for arginine synthesis; developmental changes in the expression of arginine-synthesizing and -catabolizing enzymes. BMC Dev Biol 2008, 8:107-122.

25. Wilson MD, Barbosa-Morais NL, Schmidt D, Conboy CM, Vanes L, Tybulewicz VLJ, Fisher EMC, Tavaré S, Odom DT: Species-specific transcription in mice carrying human chromosome 21. Science 2008, 322:434-438.

26. Phillips MM, Sheaff MT, Szlosarek PW: Targeting arginine-dependent cancers with arginine-degrading enzymes: opportunities and challenges. Cancer Res Treat 2013, 45:251-262.

doi:10.1186/1423-0127-21-42

Cite this article as: Shive et al.: A transgenic approach to study

argininosuccinate synthetase gene expression. Journal of Biomedical Science 2014 21:42.

\section{Submit your next manuscript to BioMed Central and take full advantage of:}

- Convenient online submission

- Thorough peer review

- No space constraints or color figure charges

- Immediate publication on acceptance

- Inclusion in PubMed, CAS, Scopus and Google Scholar

- Research which is freely available for redistribution 